\title{
BL LAC X-RAY SPECTRA: SIMPLER THAN WE THOUGHT
}

\author{
ERIC S. PERLMAN, TIMOTHY DAUGHERTY, ANURADHA KORATKAR \\ Joint Ctr for Astrophysics, University of Maryland-Baltimore County \\ GRZEGORZ MADEJSKI, KARL ANDERSSON \\ Stanford University, SLAC \\ JULIAN H. KROLIK \\ Dept. of Physics and Astronomy, Johns Hopkins University \\ HUGH ALLER, MARGO ALLER \\ Department of Astronomy, University of Michigan \\ JOHN T. STOCKE \\ Center for Astrophysics and Space Astronomy, University of Colorado \\ TRAVIS RECTOR \\ Department of Physics and Astronomy, University of Alaska - Anchorage \\ PAOLO PADOVANI \\ European Southern Observatory \\ ALAN MARSCHER \\ Department of Astronomy, Boston University \\ MARK ALLEN \\ Centre de Donnees Astronomique \\ STEFAN WAGNER \\ Landessternwarte Heidelberg
}




\begin{abstract}
We report results from XMM-Newton observations of thirteen X-ray bright BL Lacertae objects, selected from the Einstein Slew Survey sample. The spectra are generally well fit by power-law models, with four objects having hard ( $\alpha<$ $\left.1 ; F_{\nu} \propto \nu^{-\alpha}\right)$ spectra that indicates synchrotron peaks at $>5 \mathrm{keV}$. None of our spectra show line features, indicating that soft X-ray absorption "notches" must be rare amongst BL Lacs, rather than common or ubiquitous as had previously been asserted. We find significant curvature in most of the spectra. This curvature is almost certainly intrinsic, as it appears nearly constant from 0.5 to $6 \mathrm{keV}$, an observation which is inconsistent with the small columns seen in these sources.
\end{abstract}

\title{
1. Introduction
}

The nature of the X-ray emission and absorption from BL Lacs is still an open question. Most BL Lac objects have X-ray spectra that can be fit by power-laws within smaller bandpasses, such as that of $\operatorname{ROSAT}([12],[17])$ More recent results from $A S C A[7]$ and BeppoSAX ([1],[11],[19]) have generally confirmed this spectral morphology, and also added onto it the possibility of intrinisic spectral curvature across a wider bandpass $([4],[6],[9],[16])$. In addition, earlier missions had indicated that some BL Lac objects showed a deficit in soft X-rays below a power-law model, which had been interpreted by invoking X-ray absorption features at $0.5-0.8 \mathrm{keV}([3],[8],[14],[15])$. But not all bright BL Lacs were found to require such features (e.g., [5]).

\section{Sample, Observations and Data Reduction}

We selected our targets from the Einstein Slew Survey sample of BL Lacs [13]. The Slew Survey sample is the largest collection of X-ray bright BL Lacs, and was the first contain significant numbers of both HBLs and LBLs (high-energy and low-energy peaked BL Lacs; Urry \& Padovani 1995). We received time for the $13 \mathrm{X}$-ray brightest objects (all HBLs) which were not on either XMM GTO lists or in the Cycle 1 Chandra schedule. Seventeen observations were done (four were repeated due to background flares). Because the main goal was to address the class properties, our integration times were short, $\sim 5 \mathrm{ks}$. As a result, the best data come from the EPIC instruments, which are imaging spectrographs that have low-to-moderate $(R \sim 20-50)$ spectral resolution.

All source and background extraction was done in SAS v5.4.1. X-ray spectral modeling was done in XSPEC v11.0. The PN data were fit between 1.1-10.0 keV, while the MOS data were fit between 0.5-10.0 keV, except for the faintest objects which we capped at $7.0 \mathrm{keV}$ due to low count rates at the highest energies. Where multiple observations of an object were obtained, 
each observation was reduced and analyzed separately. Three models were fit: a single power law, sum of two power laws, and a logarithmic parabola. Each model was attempted with Galactic and variable absorption, and we also fitted several sub-bands to investigate curvature.

\section{Results}

The spectral indices we found range from $\Gamma=1.7-3.5$, with $12 / 17$ being in the range $\Gamma=2.0-2.9$. This is similar to previous findings. Four spectra were found to be flat $(\Gamma<2)$. These objects are likely to have $\nu_{\text {peak }}>5 \mathrm{keV}$. In 14/17 observations, a better fit was obtained by allowing for spectral curvature, which may be intrinsic, or the result of additional absorption. We believe the curvature is most likely intrinsic, because it appears nearly constant between $0.5-6 \mathrm{keV}$ in all objects where it is required. This is inconsistent with absorption given the observed columns, which range from $3-20 \times 10^{20} \mathrm{~cm}^{-2}$. The curvature can be characterized by $d \Gamma / d(\log E) \approx 0.4 \pm 0.15$.

A similar curvature was found by Giommi et al. [4] for about $50 \%$ of X-ray bright BL Lacs observed by BeppoSAX. The greater percentage we find to require curvature, is consistent with the greater sensitivity of XMM. This type of curvature has more recently been analyzed $([9],[10])$ in the context of particle acceleration models, and given the multiple emission regions that most likely contribute to BL Lac X-ray spectra, a continuous curvature is the most likely result of spectral aging in several regions with different physical parameters (as opposed to the simpler model of a sharp cutoff, which is more commonly assumed).

The finding of significant absorption features, stands in stark contrast to the claims of "ubiquitous absorption features" in the spectra of BL Lac objects, made by earlier workers. We are confident of this result based on the high signal-to-noise of these spectra. In addition, another, independent study of four of the five BL Lacs where BBXRT and ASCA spectra appeared to give these line features[2], found a similar lack of features. We believe the most consistent explanation for the earlier results is that those spectra did indeed show curvature, similar to what we see in our spectra, but interpreted that curvature incorrectly as absorption.

\section{References}

1. Beckmann, V., et al., 2002, A \& A 383, 410.

2. Blustin, A. J., Page, M. J., Branduardi-Raymont, G., 2004, A \& A 417, 61. 

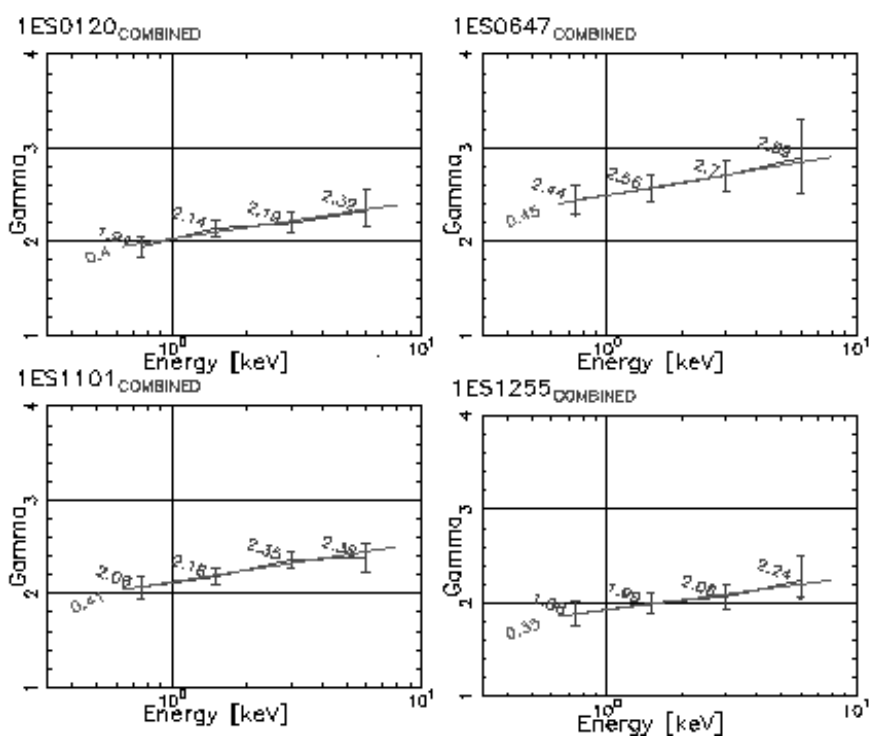

Figure 1. Four examples of the curved X-ray spectra seen for the objects in our sample. All of these objects show steeper spectra at higher energies, with curvature that remains constant through at least $6 \mathrm{keV}$.

3. Canizares, C. R., Kruper, J., 1984, ApJL, 278, L99.

4. Giommi, P., et al., 2002, in Blazar Astrophysics with BeppoSAX and Other Observatories (Rome: ESA-ESRIN), ed. P. Giommi, E. Massaro \& G. Palumbo, p. 63.

5. Guainazzi, M., et al., 1999, A \& A 342, 124.

6. Inoue, S., Takahara, F., 1996, ApJ 463, 555.

7. Kubo, H., et al., 1998, ApJ, 504, 693.

8. Madejski, G., et al., 1991, ApJ, 370, 198.

9. Massaro, E., et al., 2004, A \& A 413, 489.

10. Massaro, E., et al., 2002, in Blazar Astrophysics with BeppoSAX and Other Observatories (Rome: ESA-ESRIN), ed. P. Giommi, E. Massaro \& G. Palumbo, p.3.

11. Padovani, P., et al., 2001, MNRAS 328, 931.

12. Perlman, E. S., et al., 1996a, ApJ, 456, 451.

13. Perlman, E. S., et al., 1996b, ApJS, 104, 251.

14. Sambruna, R. M., et al., 1997, ApJ, 483, 774.

15. Sambruna, R. M., Mushotzky, R. F., 1998, ApJ, 502, 630.

16. Tavecchio, F., Maraschi, L., Ghisellini, G., 1998, ApJ, 508, 608.

17. Urry, C. M., et al., 1996, ApJ, 463, 444.

18. Urry, C. M., Padovani, P., 1995, PASP, 107, 803.

19. Wolter, A., et al., 1998, A \& A, 335, 899. 